\title{
Improvement on Bit-Error-Rate Performance of DS-CDMA by Employing 4-Elliptical Phase Shift Keying
}

\author{
Chunyi SONG, Student member, IEEE, Shigeru SHIMAMOTO, Member, IEEE
}

\begin{abstract}
Some modulation schemes with the general name of Elliptical Modulation Schemes, have been proposed and evaluated, in terms of Bit-Error-Rate (BER) performance, namely are Eccentricity Shift Keying (ESK). Inclination Angle Shift Keying (IASK), Rotation Frequency Shift Keving (RFSK) and Elliptical Phase Shift Keving (EPSK). Based on their advantages over existing modulation schemes, this paper aimed to confirm the contributions of 4-EPSK, one of Elliptical Modulation Schemes with 2-bit information transmission capability, to the improvement of CDMA communication systems. In the paper, we investigated the possible applications of 4-EPSK to CDMA systems, through evaluating BER performance of synchronous DS-CDMA by employing 4-EPSK as its modulation scheme, with an $M$-sequence and an orthogonal Gold sequence respectively, along with comparison to that of DS-CDMA by employing QPSK; simulation results have been achieved based on changeable number of users, under $A W G N$ and flat Rayleigh fading environment respectively. Advantages of DS-CDMA-4-EPSK over existing DS-CDMA-QPSK achieved in simulations, implies that by employing 4-EPSK as its modulation scheme, $C D M A$ systems can be improved in terms of BER performance.
\end{abstract}

Index Terms - BER, DS-CDMA, 4-EPSK, Number of user.

\section{INTRODUCTION}

$\mathrm{N}^{\mathrm{N}}$ addition to amplitude, frequency and phase, of a sinusoid signal used in existing modulation schemes, our proposed Elliptical Modulation Schemes [1], introduce additional attributes such as eccentricity, offset inclination angle, rotation frequency and rotation direction of an elliptical signal, through employing geometrical characteristics of an ellipse to manipulate a signal. Introduction of new modulation variables provides Elliptical Modulation Schemes with higher flexibility, and thus generation of free signals with different characteristics becomes possible, which suggests the potential applications of elliptical signals to communication systems, such as Software Defined Radio and UWB, etc. Elliptical Modulation Schemes are expected to make contributions to the improvement of wireless communication systems, through advantages over existing modulation schemes, on either BER performance or spectral efficiency (Fig. 1) [2].

This rescarch project is supported by Japanese Ministry of Science and Education Grants-in-Aid for Scientific Research, Exploratory Rescarch NO.15656094, and by Waseda University Speciai Grant.

Chunyi SONG and Shigeru SHIMAMOTO are with Graduate School of Global information and Telecommunication. Studies. Waseda University, Tokyo, 169-005I, Japan.

E-mail:_song@sl.giti.waseda.ac.jp\& shima@giti.waseda.ac.jp.

\section{Reviews of 4-Elliptical Phase Modulation SCHEMES}

As an instance of Elliptical Phase Shift Keying (EPSK), 4-EPSK has been defined through combining Inclination Angle Shift Keying (IASK) and BPSK [3]. Definitions of IASK and 4-EPSK will be reviewed in this section.

\section{A. Inclination Angle Shift Keying (IASK)}

Distinctive waveforms can be produced by changing offset inclination angles, which suggests that binary numbers can be represented by ellipses inclined at different angles. This is defined as IASK. As an instance, for two ellipses with eccentricity of 0.9 In Fig.2, waveform of the one inclined at $\pi / 4$ leans to right, while that of the one inclined at $-\pi / 4$ leans to left. These two ellipses can be used to represent binary numbers 0 and 1 respectively, and the distinction between two waveforms can be employed to distinguish two signals from each other.

IASK signals are expressed as

$$
s(t)=a \sqrt{\frac{1-e_{c}^{2}}{1-e_{c}^{2} \cos ^{2}\left(w_{r} t-\alpha_{i}\right)}} \cos w_{r} t,
$$

where a $=$ semi-major axis, $\omega_{\mathrm{r}}=$ revolution angular frequency, $\mathrm{e}_{\mathrm{c}}=$ eccentricity, $\alpha_{i}=$ offset inclination angle.

\section{B. 4-Elliptical Phase Shift Keying (4-EPSK)}

In Elliptical Modulation Schemes, EPSK was defined through combining effect of offset inclination angle and that of signal's phase. General equation of EPSK can be given out as

$$
s(t)=a \sqrt{\frac{1-e_{c}^{2}}{1-e_{e}^{2} \cos ^{2}\left(w_{r} t+\phi_{j}-\alpha_{i}\right)}} \cos \left(w_{r} t+\phi_{j}\right),
$$

where $a=$ semi-major axis, $e_{c}=$ eccentricity, $\omega_{r}=$ revolution angular frequency, $\alpha_{i}=$ offset inclination angle, and $\phi_{j}=$ signal's phase. $\alpha_{i}$ and $\phi_{j}$ can have $N$ and $M$ discrete values respectively. It should be noted that transmission capability can not be simply determined by value of $N \times M$.

As an instance, both 4-EPSK and 8-EPSK can be defined through fixing $N$ to value " 2 " and $M$ to value "4." Their differences lie in: 1) 4-EPSK was defined through combining BPSK and IASK, thus there are two phases corresponding to each inclination angle, but without repeat in values of all four 
phases; while 8-EPSK was defined through combining QPSK and IASK, and values of four phases corresponding to each inclination angle are the same; 2) 4-EPSK is capable of 2-bit information transmission under the same carrier frequency simultaneously; while 8-EPSK possesses the capability of 3-bit information transmission.

Fig. 3 is the constellation diagrams of 4-EPSK. As an example, ellipses inclined at $\pi / 4$ and $-\pi / 4$ are used to represent the first bits of 0 and 1 (the bold numbers) respectively. As we do in BPSK, another bit of 0 and 1 then can be assigned to two phases of each constellation. Thus, all 2-bit message sequences from 00 to 11 have been mapped to the two constellations.

\section{II. APPLication OF 4-EPSK to DS-CDMA SYSTEM}

In our previous jobs, advantages of 4-EPSK over QPSK in terms of BER have been achieved through Matlab simulations, under AWGN and flat Rayleigh fading respectively (Fig.4) [3]. Based on this achievement, here we are going to investigate the possible contributions of 4-EPSK to the improvement of existing popular wireless communication systerns, such as CDMA we will do this section.

Fig.5 is the outline of the simulation procedure used to investigate BER performance of synchronous DS-CDMA [4]. In synchronous DS-CDMA, users employ their own sequences to spread the information data. At each user's terminal, the information data are modulated by 4-EPSK. Then the first bits of the modulated data are spread by a code sequence, such as an M-sequence, a Gold sequence or an orthogonal Gold sequence. The spread data of all users are transmitted to the base station at the same time. The base station detects the information data of each user by correlating the received signal with a code sequence allocated to each user, and then detected data are demodulated by 4-EPSK.

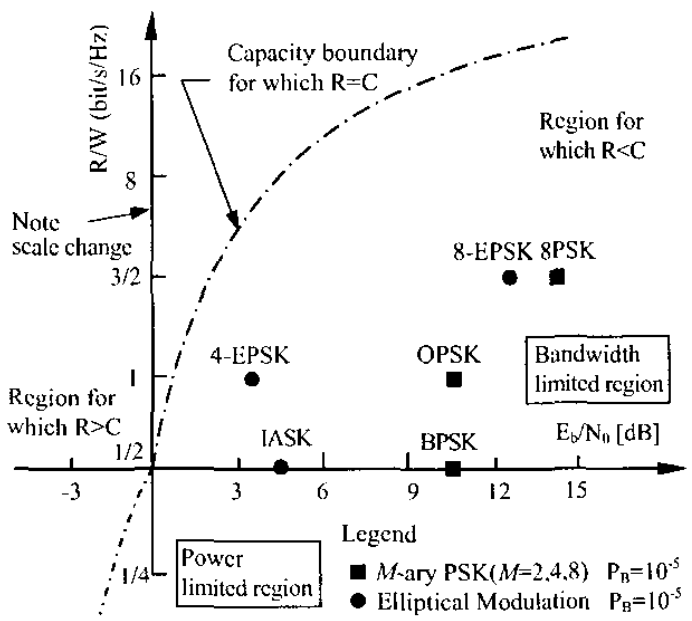

Fig.1. Bandwidth-efficiency plane. The plane has been made based on simulation results. $A$ paired Square Root Raised Cosine filters with rolloff factor $a=0.5$ were used in simulations.

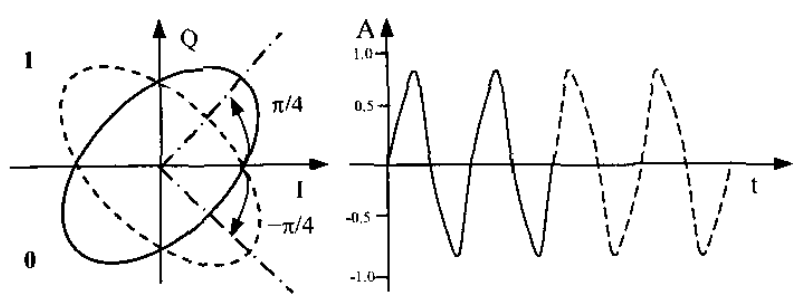

Fig.2, An example of IASK signals

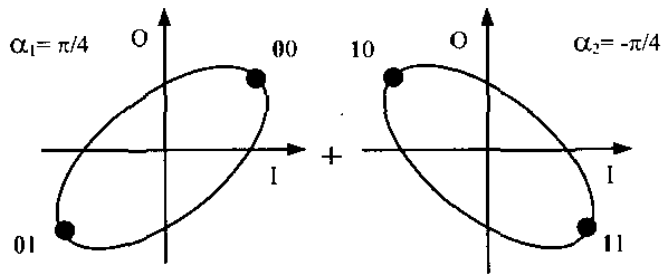

Fig.3. Constellation diagrams of 4-EPSK

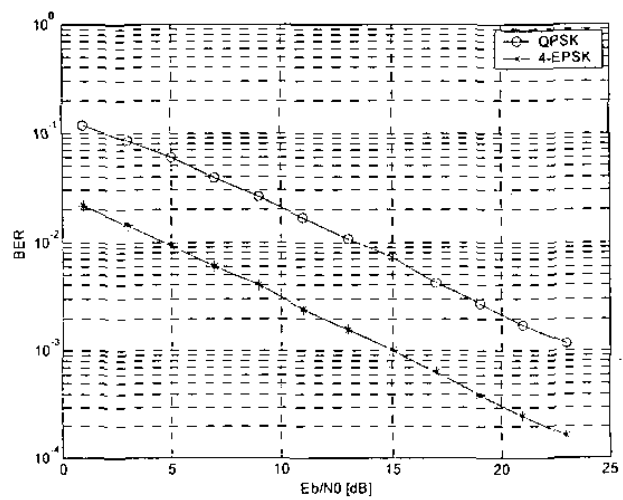

Fig.4. BER performance comparison between 4-EPSK and QPSK under one-path flat Rayleigh fading

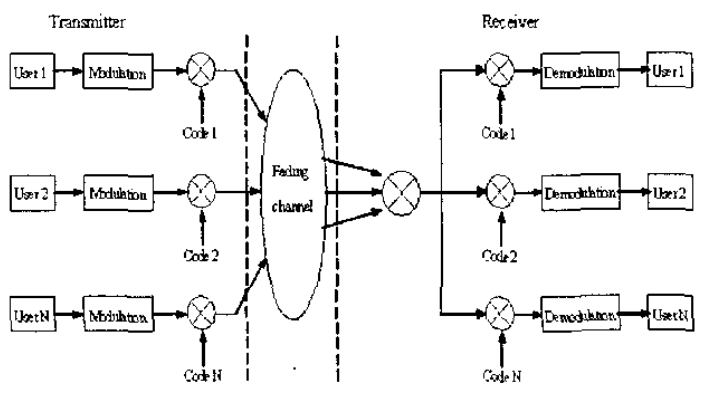

Fig.5. Simulation model used to evaluate BER performance of DS-CDMA

\section{A. Generation of code sequence}

In CDMA systems, the choice of the type of code sequence is important with respect to the resistance against both multipath interference and multi-user interference. Each code sequence must: 1) be periodic with a constant length;2) be easy to be distinguished from its time-shifted code; 3 ) be easy to be distinguished from each code sequence. 


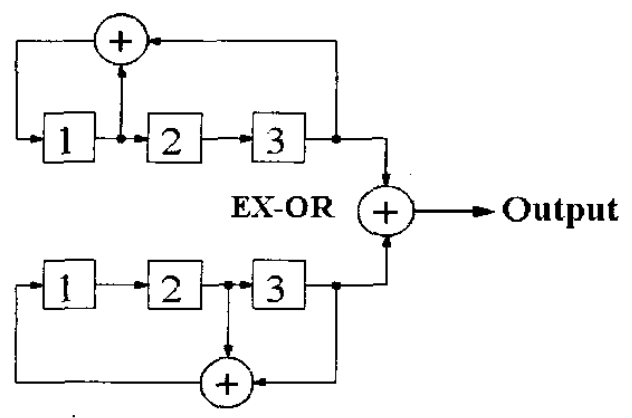

Fig,6. Three stage Feedback Shift Register for Gold sequence generation

There are several ways to generate code sequences, and here the code sequence was generated by using Feedback Shift Registers (FSRs), which is generally used in CDMA systems [4]. In Fig.6. binary bits are shifted through the different stages of register. The output of the last stage and the output of the intermediate stage are combined and fed as input to the first stage. The register starts with an initial sequence of bits, or initial state, stored in its stages. Then the register is blocked, and bits are moved through the stages. Through this way, the register continues to generate output bits and feed input bits to its first stage [5].

In the simulations, A three stage linear FSRs has been employed to generate an M-sequence at first; A Gold sequence was then generated by exclusive OR (EXOR) of two Msequences, whose relationship is that of a preferred pair; Through adding one chip to a Gold sequence to balance the proportion of 0 to 1 , the Gold sequence comes into being an orthogonal Gold sequence.

An M-sequence has good autocorrelation. However, the number of sequences that have the same code length and the same correlation characteristics is limited. A Gold sequence has many different codes in comparison with an $\mathrm{M}$-sequence, while it has the problems such as: 1) the proportion of 0 and 1 is not always balanced; 2 ) the cross-correlation value is not 0 in a synchronized environment; 3 ) the code length is an odd number. Through adding one chip to a Gold sequence to balance the proportion of 0 and 1 , an orthogonal Gold sequence has 0 cross-correlation value in a synchronized environment.

\section{B. Simulation Model of Transmitter}

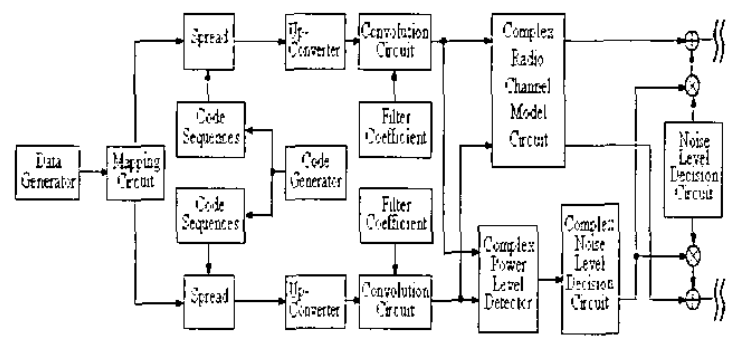

Fig 1. Smalation mode of tran maiter
According to definition of 4-EPSK in section II, through substituting $\left(\alpha_{1}=\pi / 4, \phi_{1}=\pi / 4\right),\left(\alpha_{1}=\pi / 4, \phi_{2}=5 \pi / 4\right),\left(\alpha_{2}=-\right.$ $\left.\pi / 4, \phi_{3}=-\pi / 4\right)$, and $\left(\alpha_{2}=-\pi / 4, \phi_{4}=3 \pi / 4\right)$ to (2), four 4-EPSK modulated signals can be obtained as:

$$
\begin{aligned}
& s\left(\alpha_{1}, \phi_{,}, t\right)=a \sqrt{\frac{1-e_{c}^{2}}{1-e_{c}^{2} \cos ^{2}\left(w_{r} t+\pi / 4-\pi / 4\right)}} \cos \left(w_{r} t+\pi / 4\right) \\
& =a \sqrt{\frac{1-e_{c}^{2}}{1-e_{e}^{2} \cos ^{2} w_{r} t}} \cos \left(w_{r} t+\pi / 4\right) \\
& s\left(\alpha_{1}, \phi_{2}, t\right)=a \sqrt{\frac{1-e_{c}^{2}}{1-e_{c}{ }^{2} \cos ^{2}\left(w_{r} t+5 \pi / 4-\pi / 4\right)}} \cos \left(w_{r} t+5 \pi / 4\right) \\
& =a \sqrt{\frac{1-e_{c}^{2}}{1-e_{c}^{2} \cos ^{2} w_{r} t}} \cos \left(w_{r} t+\pi / 4\right) \\
& s\left(\alpha_{2}, \phi_{3}, t\right)=a \sqrt{\frac{1-e_{c}^{2}}{1-e_{c}^{2} \cos ^{2}\left[\left(w_{r} t-\pi / 4-(-\pi / 4)\right]\right.}} \cos \left(w_{r} t-\pi / 4\right), \\
& =a \sqrt{\frac{1-e_{c}^{2}}{1-e_{c}^{2} \cos ^{2} w_{r} t}} \cos \left(w_{r} t-\pi / 4\right) \\
& \begin{aligned}
s\left(\alpha_{2}, \phi_{4}, t\right) & =a \sqrt{\frac{1-e_{c}^{2}}{1-e_{c}^{2} \cos ^{2}\left[\left(w_{r} / t+3 \pi / 4-(-\pi / 4)\right]\right.}} \cos \left(w_{r} t+3 \pi / 4\right) \\
& =a \sqrt{\frac{1-e_{c}^{2}}{1-e_{c}^{2} \cos ^{2} w_{r} t} \cos \left(w_{r} t+3 \pi / 4\right)} .
\end{aligned}
\end{aligned}
$$

For an easy explanation, ich and qch were used to express the 4-EPSK data transmitted in in-phase channel and quadrature-phase channel respectively.

Define the code sequence generated in section A as "code", 4-EPSK data were multiplied by the code sequence used to spread the transmitted data as:

$[i c h l, q c h l]=$ spread $[i c h, q c h$, code $] . \quad \%$ spreading

The spreaded data $i c h I$ and $q c h l$ were then oversampled and filtered by a paired Square Root Raised Cosine (SRRC) filter with roll-off factor $\alpha=0.5$.

The transmitted signals from four users were synthesized and then contaminated by AWGN and flat Rayleigh fading respectively. In reality, transmitted signals are contaminated by distinctive Rayleigh fading. To simplify the simulation model, we supposed that the synthesized signals were contaminated by Rayleigh fading (by using average values).

\section{Simulation Model of Receiver}

At the receiver, the noise-corrupted signals were filtered by using a squared SRRC filter at first. After being synchronized and resampled, the transmitted data of four users were detected by correlating the received data with the spread code used at the transmitter as: 
$\left[r_{-} i c h, r_{-} q c h\right]=$ despread $[i c h n, q c h n$, code $] ; \%$ despreading

Finally, the correlated data were demodulated by $4-E P S K$, which consists of two steps: the first bit can be determined by detecting which ellipse has been used to send out the information; and then another bit can be identified by using phase-matching. Thus, we combined correlation detector with coherent demodulator in the receiver (Fig.8) [6] [7] [8].

\section{1) Detection of IASK Signals}

In our previous jobs, we found that the increase in eccentricity can contribute to the improvement of IASK performance in terms of BER. Thus, two IASK carrier signals with eccentricity of 0.9 have been used in simulation.

Base on their distinctive waveforms showed in Fig.2, detection of IASK signals was accomplished through comparing the waveform similarities of received signals and their replica signals [3]. As Correlations of reference signals and the received signal determine the waveform similarity or dissimilarity between each pair of signals, and $\mathrm{I} / \mathrm{Q}$ transmission has been used in the simulation model, here we combined correlation detector and $\mathrm{I} / \mathrm{Q}$ demodulator to complete this detection procedure.

Four unit signals come from transmitter to receiver through l/Q channels, which means eight correlators and eight reference signals will be needed in correlation detector. Note that the only difference between (7) and (8), and that between (9) and (10), lies in the phase. Phase shift within each pair of signals is " $\pi$ ", and $\cos \left(w_{r} t+\pi\right)=-\cos \left(w_{r} t\right), \sin \left(w_{r} t+\pi\right)=-\sin \left(w_{r} t\right)$. Thus, by adding squaring operation block, for each ellipse, we can select one signal from two, and make its replica as reference signals for both. Based on this analysis, we reduced number of correlator eight to four in simulations. In Fig.9 [9], the upper two channels were configured to detect two unit signals of ellipse inclined at $\pi / 4$, while the lower two branches were configured to detect two unit signals of ellipse inclined at $-\pi / 4$.

Without losing generality, we selected the signal with phase of $\pi / 4$ as reference signals for the signals generated from ellipse inclined at $\pi / 4$, whose phase is either $\pi / 4$ or $5 \pi / 4$; and the one with phase of $-\pi / 4$ as reference signals for the signals of ellipse inclined at $-\pi / 4$, whose phase is either $-\pi / 4$ or $3 \pi / 4$. Four $\mathrm{I} / \mathrm{Q}$ modulated reference signals then can be expressed as [10]:

$$
\begin{aligned}
& r e f \__{-} i 1=a \sqrt{\frac{1-e_{r}^{2}}{1-e_{c}{ }^{2} \cos ^{2} w_{r} t}} \cos \left(w_{r} t+\pi / 4\right) \cos w_{0} t \\
& r_{-} q 1=a \sqrt{\frac{1-e_{c}^{2}}{1-e_{c}^{2} \cos ^{2} w_{r} t}} \sin \left(w_{r} t+\pi / 4\right) \sin w_{v} t \\
& r e f_{-} i 2=a \sqrt{\frac{1-e_{c}{ }^{2}}{1-e_{c}{ }^{2} \cos ^{2} w_{r} t}} \cos \left(w_{r} t-\pi / 4\right) \cos w_{0} t \\
& r e f_{-} q 2=a \sqrt{\frac{1-e_{c}^{2}}{1-e_{c}^{2} \cos ^{2} w_{r} t}} \sin \left(w_{r} t-\pi / 4\right) \sin w_{0} t
\end{aligned}
$$

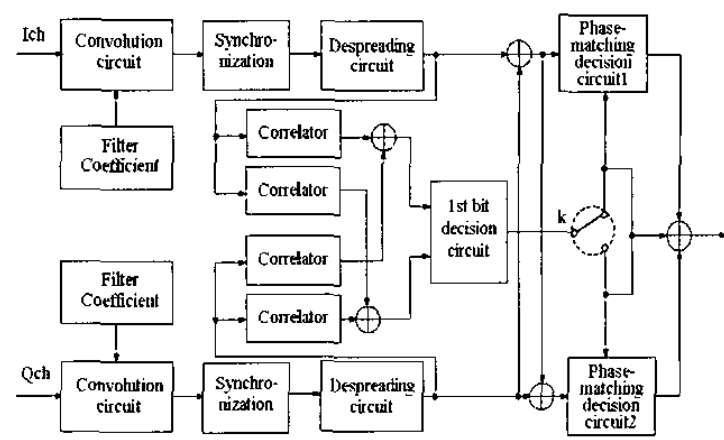

Fig.8. Simul zxtion modet of rectiver

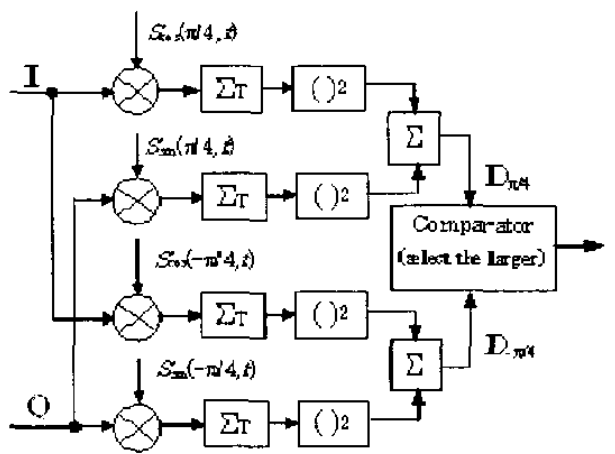

Fig 9. Combined receiver of $U / Q$ demo dulator and correhtion detecto $r$

Four Correlators calculate correlations between reference signals and the received signal, which stands for the waveform similarity or dissimilarity between each pair of signals:

$$
\begin{aligned}
& c_{i 1}(t)=r_{-} i c h \times r e f_{-} i 1, \\
& c_{q 1}(t)=r_{-} q c h \times r e f \_q 1, \\
& c_{i 2}(t)=r_{-} i c h \times r e f_{-} i 2, \\
& c_{q 2}(t)=r_{-} q c h \times r e f_{-} q 2 .
\end{aligned}
$$

Then within one sampling period T,

$$
\begin{aligned}
& C_{{ }_{1}}(T)=\sum_{t_{0}}^{t_{0}+T} c_{i 1}(t), \\
& C_{Q 1}(T)=\sum_{t_{0}}^{t_{0}+T} c_{q 1}(t), \\
& C_{I 2}(T)=\sum_{t_{0}}^{t_{0}+T} c_{i 2}(t), \\
& C_{Q 2}(T)=\sum_{t_{0}}^{t_{0}+T} c_{42}(t) .
\end{aligned}
$$

In Fig.9, the blocks following the product integrators perform a squaring operation to prevent appearance of negative values. Thus, the two signals having phase shift of $\pi$ and two replica signals can have the same correlation values. 
As we know, for each pair of signals, the bigger the similarity, the larger the correlation. Therefore, the received signal was determined corresponding to the larger decision variable of:

$$
\begin{aligned}
& D_{\pi / 4}=\left[C_{I 1}(T)\right]^{2}+\left[C_{Q 1}(T)\right]^{2}, \\
& D_{-\pi / 4}=\left[C_{12}(T)\right]^{2}+\left[C_{Q 2}(T)\right]^{2} .
\end{aligned}
$$

If $D_{\pi / 4}>D_{-\pi / 4}$, correlation detector judges that the elliptical carrier inclined at $\pi / 4$ has been used to send out the signal, and thus the first bit of received signal is " 0 ";

If $D_{-\pi / 4}>D_{\pi / 4}$, correlation detector judges that the elliptical carrier inclined at $-\pi / 4$ has been used to send out the signal, and then the first bit of received signal is " 1 ";

\section{2) Detection of BPSK Signals}

Demodulation of the second bit of received signal is quite similar with BPSK. Different point lies in: for BPSK, the decision region is known to us before transmission; while in the case of 4-EPSK, decision regions of phase-matching for two ellipses are different, thus, it can be decided only after identifying which ellipse has been used to send out the first bit information. In Fig.8, " $\mathrm{K}$ " is responsible for selecting one from two phase-matching decision circuits. When first bit is determined as " 0 ," which means ellipse inclined at $\pi / 4$ has been used to send out the information, then phase-matching decision circuitl will be connected and activated, and its decision regions of phase-matching are $[-\pi / 4,3 \pi / 4]$ and $[3 \pi / 4$, $7 \pi / 4]$ respectively; while decision regions change to $[-3 \pi / 4$, $\pi / 4]$ and $[\pi / 4,5 \pi / 4]$, when phase-matching decision circuit2 is connected and activated due to the detected first bit is " 1. ."

Coherent demodulation was used here. As received data are $\mathrm{fl} / \mathrm{Q}$ data, based on assumption of perfect synchronization, phase of received signal can be calculated as:

$$
\phi(T)=\operatorname{angmat}\left[r_{-} i c h\left(t_{0}\right)+j \times r_{-} i c h\left(t_{0}\right)\right],
$$

where angmat is Matlab's built-in function, and " $\mathrm{t}_{0}$ " stands for the first unit time in each sampling period.

Suppose that the ellipse inclined at $\pi / 4$ has been used to send out the information, then output of demapping circuit is: 1) if $\not(T) \in[-\pi / 4,3 \pi / 4]$, the received signal is decided to be [00];

2) if $\phi(T) \in[3 \pi / 4,7 \pi / 4]$, the received signal is decided to be [01].

\section{Results AND Conclusions}

In synchronous DS-CDMA, the number of code sequences that can be allocated to different users is equal to the number of code length [10]. Therefore, the number of users must be smaller than the length of code sequence. The length of code sequence used here is " 8 ," thus, the number of user can be set to be a changeable value from 1 to 7 . Without losing generality, in Matlab simulations, the number of user has been set to be 1,4 , and 7 respectively.

BER performance of synchronous DS-CDMA by employing 4-EPSK has been evaluated, with an M-sequence and with an orthogonal Gold sequence respectively, along with comparison to that of DS-CDMA by employing QPSK as its modulation scheme.

Fig. 10 showed that increase in the number of user leads to the worse performance of DS-CDMA, due to worse multi-user interference. It can also be explained from mathematical point view: cross-correlation of an $\mathrm{M}$-sequence is not 0 in a synchronized environment, and these non-zero correlations become interference for other users; therefore, the more the user, the worse the multi-user interference, and the worse the performance. In contrast, in the case when an orthogonal Gold sequence is employed, the value of cross-correlation is 0 in perfect synchronized environment. Thus, increase in the number of user will lead BER performance of DS-CDMA approach to the theoretical values.

In simulations, DS-CDMA by employing 4-EPSK has shown better performance than that by employing QPSK (Fig.10, Fig.11), which implies that CDMA systems can be improved in terms of BER, by employing 4-EPSK as its modulation scheme.

\section{ACKNOWLEDGMENT}

Authors thank Denki-Tuusin-Fukyuu-Zaidan of Japan for their financial support.

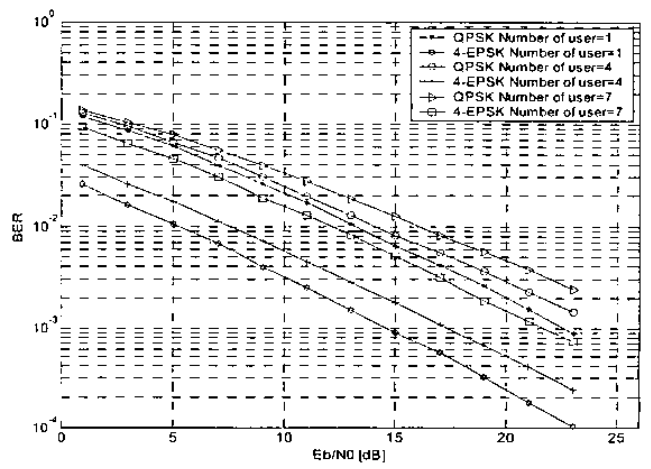

Fig.10. BER performance comparison between CDMA-QPSK and CDMA-4-EPSK with an M-sequence. Number of user were set to be 1, 4 and 7 , respectively

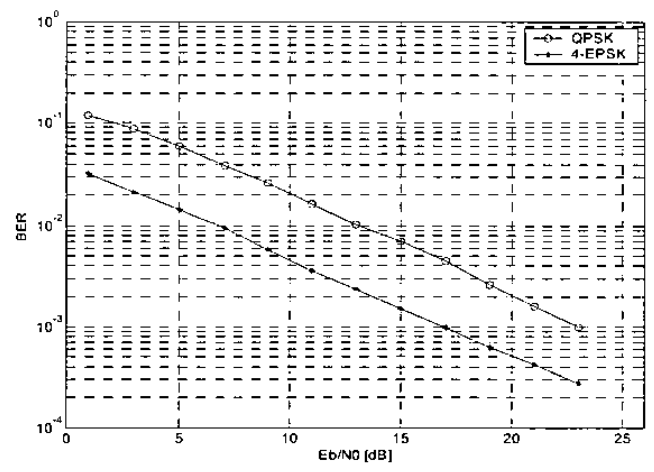

Fig.l1. BER performance comparison between CDMA-QPSK and CDMA-4-EPSK with an orthogonal Gold sequence, where number of user was set to be 7 


\section{REFERENCES}

[1] S.Shimamoto, "Proposal of new modulation scheme cmploying elliptical circle". IEEE Wireless Communications and Networking Conference 2003. March 2003, New Orleans, USA.

[2] B. Sklar, "Defining, Designing, and Evaluating Digital Communication Systcms, "IEEE Commun. Mag.. vol.31, no.11, ,pp.92-101, Nov.1993

[3] Churyi Song and S.Shimamoto, "Evaluations of Elliptical Modulation Scheme". IEEE Wireless Communications and Networking Conference 2004. March 2004, Atlanta, Georgia USA.

[41 Hiroshi Harada and Ramjec Prasad, "Configuration of a Transmitter and a Receiver by using a computer program," in Sinulation and Software Radio, London: Artcch House, 2002,.pp.244-249.
[5] Samuel C.Yang,"Multiple Access," in CDMA RF System Engineering, Artech House, 1998, pp.43-58.

[6] B.P.Lathi, Modern Digital and Analog Commanication System, Oxford University Press, Oxford, 1998.

[7] S.Rappaport, "Binary Phase Shift Keying" in Wireless CommunicationsPrinciples and Practice (Second Edition), Prentice Hall, 2002, pp295-pp298.

[8] Alister Burr, "Modem Design" in Modulation and Coding for Wireless Communications, Prentice Hall, 2001, pp77-pp109.

[9] B.Sklar, "Binary Differential PSK Examplc," in Digital Communications-Fundamentals and Applications ( Second Edition ). Prentice Hall: New Jersey, 2001, pp.196-200.

[10] Alister Burr, "The Complex Bascband Representation" in Modulation and Coding for Wireless Communications. Prentice Hall, 2001, pp 16-ppl9. 